\title{
Processos judiciais em oftalmologia: análise de possíveis fatores desencadeantes
}

\author{
Litigation in ophthalmology: analysis of possible triggers
}

Wellington Santos ${ }^{1}$, Helena Parente Solari ${ }^{2}$, Marcelo Palis Ventura²

\section{RESUMO}

Objetivo: Analisar os possíveis fatores de desencadeamento de processos judiciais na área de oftalmologia.

Método: Estudo retrospectivo de 70 casos de processos judiciais cíveis em oftalmologia. Foram avaliados em cada processo, a anamnese, o exame oftalmológico, os dados do paciente no momento do incidente, sexo, idade, diagnóstico inicial e diagnóstico da causa do processo, número de consultas antes eapós o fato desencadeante, a realização de exames pré-operatórios mínimos de acordo com os protocolos da Cooperativa Estadual de Serviços Administrativos em Oftalmologia - COOESO, termo de consentimento informado, as folhas de descrição do procedimento, de enfermagem, de materiais e medicamentos do centro cirúrgico, do anestesista, da alta hospitalar e o valor da indenização.

Resultados: Dos 70 casos analisados, com relação à acuidade visual final, $45(64,3 \%)$ apresentavam amaurose. Quanto às condições prévias ao tratamento, os diagnósticos foram: catarata $46(65,7 \%)$, ametropias $12(17,1 \%)$, trauma ocular $8(11,4 \%)$, glaucoma $3(4,3 \%)$ e descolamento de retina $1(1,4 \%)$. Com relação às principais complicações secundárias ao tratamento efetuado que deram motivação à lide, os diagnósticos foram: descolamento de retina $37(52,8 \%)$, acuidade visual insatisfatória pós cirurgia de catarata $12(17,1 \%)$, irregularidades corneanas após cirurgia refrativa $8(11,4 \%)$, endoftalmites $4(5,7 \%)$, desconforto com óculos prescritos $4(5,7 \%)$ e atrofia bulbar $4(5,7 \%)$. O número de consultas antes do início do processo foi de até duas em $67,1 \%$ dos casos. Os processos decorrentes de cirurgia representaram 94,3\% da amostra. Termo de consentimento informado não foi utilizado em 63\% do total de casos cirúrgicos. Os valores de indenização pagos foram menores do que 50 mil reais em $72,7 \%$.

Conclusões: Foram fatores importantes relacionados aos processos judiciais oftalmológicos: amaurose, tratamento cirúrgico, descolamento de retina, número reduzido de consultas pré-operatórias e ausência de termo de consentimento.

Descritores:Imperícia;Consentimento livre eesclarecido; Oftalmologia/legislação \& jurisprudência; Responsabilidade legal; Procedimentos cirúrgicos refrativos/legislação \& jurisprudência

\begin{abstract}
Purpose: To analyze the possible predisposing factors of lawsuits involving ophthalmologists.

Methods: Retrospective study of 70 lawsuits involving the practice of Ophthalmology. The patient's gender, age, medical and ophthalmic history were reviewed. The eye disorder that generated the prosecution, the pre-existing ophthalmic diagnosis, the preoperative exams and the operating room data were also analyzed. The informed consent form, the outcome of the litigation and the value of compensation were recorded.

Results: Blindness was reported in 45 (64.3\%) of the 70 lawsuits and was the principal eye disorder related to litigation. The pre-existing eye diseases associated with legal claims were: cataracts in 46 (65.7\%) lawsuits, refractive errors in $12(17.1 \%)$, ocular trauma in $8(11.4 \%)$, glaucoma in $3(4.3 \%)$ and retinal detachment in 1 (1.4\%) lawsuit. The major surgical complications that possibly motivated the patient to sue the ophthalmologist were retinal detachment in 37 (52.8\%) lawsuits, poor visual acuity after cataract surgery in 12 (17.1\%), corneal disorders following refractive surgery in 8 (11.4\%), endophthalmitis in $4(5.7 \%)$, discomfort with refractive prescriptions in $4(5.7 \%)$ and ocular atrophy in 4 (5.7\%) lawsuits. Ophthalmic surgery was involved in $94.3 \%$ of the 70 prosecutions analyzed. Two or less visits to the ophthalmologist preceding the surgical or refractive event leading to legal dispute were recorded in $67.1 \%$ of the lawsuits. A pre-surgical Informed consent form was not used in $63 \%$ of the 66 surgical procedures leading to litigation. $62.9 \%$ of the lawsuits were unfavorable to the Ophthalmologist and the amount of compensation was less than $R \$ 50,000$ in $72.7 \%$ of these legal claims.

Conclusions: Blindness, surgery, retinal detachment, few pre-operative ophthalmic visits and the lack of informed consent forms were possible risk factors associated with lawsuits in the field of Ophthalmology.
\end{abstract}

Keywords: Malpractice; Informed consent; Ophthalmology/legislation \& jurisprudence; Liability, legal; Refractive surgical procedures/legislation \& jurisprudence
Trabalho realizado na Universidade Federal Fluminense, Rio de Janeiro.

${ }^{1}$ Médico, Ciências Médicas, Universidade Federal Fluminense - UFF - Rio de Janeiro (RJ) Brasil.

Médico, Departamento de Oftalmologia, Universidade Federal Fluminense - UFF - Rio de Janeiro (RJ), Brasil.

Endereço para correspondência: Marcelo Palis Ventura. Rua Mariz e Barros, 459 Niterói (RJ) - CEP 24220-120 - E-mail: palis@infolink.com.br

Trabalho realizado como parte dos requisitos necessários para obtenção do grau de Mestre em Ciências Médicas da Faculdade de Medicina da Universidade Federal Fluminense.

Recebido para publicação em 25.04.2010

Última versão recebida em 07.11.2010

Aprovação em 15.11.2010

Nota Editorial: Depois de concluída a análise do artigo sob sigilo editorial e com a anuência do Dr. Carlos Teixeira Brandt sobre a divulgação de seu nome como revisor, agradecemos sua participação neste processo.

\section{INTRODUÇÃO}

0 cenário atual do exercício de medicina no Brasil apresenta um quadro que chama a atenção e os desafios a serem enfrentados não se comparam com aqueles que as gerações anteriores de médicos tiveram de lidar. Nos últimos anos assistiu-se a um expressivo aumento do número de Escolas de Medicina, algumas criadas para atender finalidades empresariais e não para atender às demandas sociais. Além disso, observou-se a consolidação das empresas conhecidas como "planos de saúde" no sistema de atendimento médico. O progresso tecnológico foi fantástico, mas ao mesmo tempo em que ampliou as condições de melhores diagnósticos e curas, multiplicou severamente os custos dos cuidados com a saúde ${ }^{(1)}$. E, ao que parece, copiou-se o modelo Norte-Americano da indústria de ações judiciais contra médicos, já que a Medicina assim o permite, por ser ciência imprecisa, de dúvidas e incertezas.

O número de processos judiciais cíveis na área médica tem aumentado muito nos últimos 10 anos. Na oftalmologia, as áreas 
em que mais têm ocorrido processos são as das subespecialidades de catarata e cirurgia refrativa(2-4).

Apesar do aprimoramento das técnicas e do avanço do conhecimento, os médicos não se inteiraram dos progressos sociais e dos direitos do paciente, hoje chamado de consumidor, pela Lei 8.078/90, onde o médico é considerado prestador de serviços, embora o novo Código de Ética Médica - Resolução CFM 1931/2009, discorde desta denominação(5). A descrição exígua nos prontuários, aliada ao fato de pouco se esclarecer ao paciente sobre os riscos inerentes dos procedimentos, na "Era da Informação", têm permitido o aumento da incidência de processos ${ }^{(6-8)}$

O objetivo deste estudo foi analisar os possíveis fatores desencadeantes de processos judiciais na área de oftalmologia.

\section{MÉTODOS}

Estudo retrospectivo de 70 casos de processos judiciais cíveis que envolveram médicos oftalmologistas, baseados em perícias realizadas no período compreendido entre 2002 e 2008, no Estado do Rio de Janeiro. Este estudo foi aprovado pelo Comitê de Ética em Pesquisa da Faculdade de Medicina da Universidade Federal Fluminense.

Os critérios de inclusão foram: processos médicos oftalmológicos transitados em julgado, com autorização por escrito dos juízes consultados. Foram excluídos aqueles em que inexistia o prontuário do paciente nos autos do processo.

Foram avaliados em cada processo os dados demográficos dos pacientes no momento do incidente, a anamnese, o exame oftalmológico, o diagnóstico inicial, o tratamento realizado, o diagnóstico da causa do processo, o número de consultas antes e após o fato desencadeante, a realização de exames pré-operatórios mínimos de acordo com os protocolos da Cooperativa Estadual de Serviços Administrativos em Oftalmologia (COOESO) $)^{(9)}$, termo de consentimento informado, foIha de descrição do procedimento, folha de enfermagem, folha de materiais e medicamentos do centro cirúrgico, folha do anestesista, folha de alta hospitalar e o valor da indenização (abaixo de 50 mil reais, de 50 mil até 100 mil reais, acima de 100 mil reais). A analise dos dados foi realizada em conjunto com o Departamento de Estatística da Universidade Federal Fluminense

\section{RESULTADOS}

Dos 70 casos analisados 27 (38,6\%) eram de mulheres e $43(61,4 \%)$ eram de homens. As idades variaram de dois meses a 83 anos, com média de 46,7 anos e desvio padrão de 18,5 anos.

Os dados da acuidade visual inicial e final estão descritos na tabela 1. Com relação ao motivo da consulta inicial os resultados encontrados foram: 46 casos de catarata (65,7\%), 8 casos de cirurgia refrativa, 8 casos de trauma ocular, 4 casos de prescrição de óculos, 3 casos de glaucoma e 1 caso de descolamento de retina. Destes, $66(94,3 \%)$ pacientes foram submetidos a tratamento cirúrgico e $4(5,7 \%)$ a tratamento clínico. Os procedimentos realizados e as complicações decorrentes que deram motivação a lide estão detalhados nas tabelas 2 e 3 .

O número de consultas realizadas antes do procedimento que deu origem ao processo foi de até duas em 47 pacientes $(67,1 \%)$ e mais de duas em 23 pacientes (32,9\%). O número de consultas após o procedimento desencadeante foi de até duas em 23 (32,9\%) pacientes e mais de duas em 47 (67,1\%).

Com relação às condições dos exames pré-operatórios de acordo com os protocolos da Cooperativa Estadual de Serviços
Tabela 1. Acuidade visual inicial e final com correção

\begin{tabular}{lcc}
\hline Acuidade visual & $\begin{array}{c}\text { Acuidade visual } \\
\text { inicial (n 70) }\end{array}$ & $\begin{array}{c}\text { Acuidade visual } \\
\text { final (n 70) }\end{array}$ \\
\hline $20 / 40$ ou melhor & $39(55,7 \%)$ & $20(28,6 \%)$ \\
$20 / 60$ a 20/400 & $23(32,9 \%)$ & $5(7,1 \%)$ \\
Amaurose & $1(1,4 \%)$ & $45(64,3 \%)$ \\
Não avaliada & $7(10,0 \%)$ & Zero \\
\hline
\end{tabular}

\begin{tabular}{lc}
$\begin{array}{l}\text { Tabela 2. Procedimentos realizados que deram } \\
\text { origem aos processos }\end{array}$ \\
\hline Tipo de procedimento & Número de casos (\%) \\
\hline Facectomia & $46(65,7)$ \\
Cirurgia refrativa & $8(11,4)$ \\
Cirurgia de trauma ocular & $8(11,4)$ \\
Prescrição de óculos & $4(5,7)$ \\
Cirurgia de glaucoma & $3(4,3)$ \\
Descolamento de retina & $1(1,4)$ \\
\hline
\end{tabular}

Tabela 3. Complicações decorrentes do procedimento realizado

\begin{tabular}{lc}
\hline Complicações & Número de casos (n 70) (\%) \\
\hline Descolamento de retina & $37(52,8)$ \\
$\begin{array}{l}\text { Acuidade visual insatisfatória } \\
\text { após cirurgia de catarata }\end{array}$ & $12(17,1)$ \\
Alterações corneanas & $8(11,4)$ \\
após cirurgia refrativa & \\
Endoftalmites & $4(5,7)$ \\
Desconforto com óculos prescritos & $4(5,7)$ \\
Atrofia bulbar & $4(5,7)$ \\
$\begin{array}{l}\text { Acuidade visual insatisfatória } \\
\text { após cirurgia de glaucoma }\end{array}$ & $1(1,4)$ \\
\hline
\end{tabular}

Administrativos em Oftalmologia - COOESO, os dados dos prontuários foram classificados como satisfatórias em 42 (63,6\%), insatisfatórias em 22 (33,3\%) e inexistentes em 2 (3\%).

Os resultados relativos à documentação pertinente ao ato cirúrgico foram os seguintes: termo de consentimento estava presente em 25 (37,9\%) e ausente em 41 (63,1\%); descrição cirúrgica dos procedimentos realizados foi adequada em 54 (81,8\%), inadequada em 9 (13,6\%) e ausente em $3(4,6 \%)$; folha de descrição de material cirúrgico estava adequada em 58 (87,9\%), inadequada em 6 (9,1\%) e ausente em 2 (3\%); folha de anestesia estava adequada em 50 (75,8\%), inadequada em $7(10,6 \%)$ e ausente em 9 (13,6\%); e a folha de alta hospitalar estava adequada em 58 (87,9\%), inadequada em $4(6,1 \%)$ e ausente em $4(6,1 \%)$.

Do total de 70 processos estudados $44(62,9 \%)$ resultaram em indenização para os pacientes e $26(37,1 \%)$ processos não resultaram em indenização. Dentre os casos em que houve indenização $32(72,7 \%)$ foram com valores menores que $\mathrm{R} \$ 50$ mil reais, $10(22,7 \%)$ com valores entre 50 mil e 100 mil reais e $2(4,5 \%)$ com valores maiores que 100 mil reais.

O quadro 1 apresenta de forma resumida a correlação entre os resultados das sentenças judiciais e os principais parâmetros desfavoráveis ao médico. 
Quadro 1. Resultados das sentenças judiciais e suas relações com os principais parâmetros desfavoráveis ao médico

\begin{tabular}{lcc}
\hline $\begin{array}{l}\text { Parâmetros } \\
\text { desfavoráveis }\end{array}$ & $\begin{array}{c}\text { Sentença } \\
\text { desfavorável ao } \\
\text { médico (n 44) }\end{array}$ & $\begin{array}{c}\text { Sentença } \\
\text { favorável ao } \\
\text { médico (n 26) }\end{array}$ \\
\hline Amaurose & 44 & 1 \\
Tratamento cirúrgico & 44 & 21 \\
$\begin{array}{l}\text { Ausência termo } \\
\text { de consentimento }\end{array}$ & 41 & 1 \\
$\begin{array}{l}\text { Procedimento inicial } \\
\text { evoluindo para } \\
\text { descolamento de retina }\end{array}$ & 37 & Zero \\
$\begin{array}{l}\text { Até } 2 \text { consultas antes do } \\
\text { fato desencadeante }\end{array}$ & 36 & 3 \\
\hline
\end{tabular}

\section{DISCUSSÃo}

Estatísticas recentes apontam para um aumento expressivo no número de processos contra médicos no Brasil. Dentre as causas deste aumento podemos citar as mudanças nas características do brasileiro como consumidor, estando mais consciente e exigente. Estas mudanças têm relação com um processo semelhante ao ocorrido nos Estados Unidos, descrito como "indústria do erro médico", com julgamentos envolvendo grandes cifras, gerando mudanças na relação médico-paciente ${ }^{(6,10-11)}$.

Em processos médicos, os juízes se baseiam nas informações fornecidas por peritos, dando veredictos a respeito de assuntos que vão além de seu campo de conhecimento. Os peritos que participam destes processos podem emitir laudos muito distantes da realidade científica, prejudicando a classe médica, portanto a existência de protocolos e normatizações específicos pode ajudar a minimizar a subjetividade dos pareceres ${ }^{(12)}$.

Através da análise de processos médicos oftalmológicos, este estudo determinou as principais características relacionadas aos médicos, aos pacientes e às condições oftalmológicas que geraram as ações litigiosas. Múltiplos fatores de risco foram associados a um aumento da probabilidade de processo médico.

Diversos estudos analisaram as principais características de casos que evoluíram para processos judiciais. A literatura demonstra que as causas mais comuns têm associação com cirurgias, sendo a catarata o principal diagnóstico, mas também apontam outras causas como as cirurgias refrativas e de glaucoma. A maior parte dos casos decorre de resultados insatisfatórios e não de erro médico(10,13-14).

Em nosso estudo, esta insatisfação pode ser claramente identificada quando comparamos a acuidade visual inicial que era de 20/40 ou melhor em 55,7\% dos pacientes, evoluindo para amaurose em $64,3 \%$, indicando que o resultado visual tem papel importante como fator de risco.

Em concordância com dados da literatura, dentre as condições que levaram à consulta inicial, os quadros relacionados à cirurgia correspondiam à maior parte dos casos, sendo a catarata a causa mais comum $(65,7 \%)$, seguida de casos com indicação de cirurgia refrativa $(11,4 \%)$, enquanto ametropias, trauma ocular, glaucoma e descolamento de retina, foram encontrados como fatores desencadeantes de processos médicos em menor escala. Dados recentes indicam que a incidência de processos judiciais tem aumentado, principalmente para procedimentos cirúrgicos eletivos. Apesar das cirurgias refrativas serem apontadas como causa crescente de queixas judiciais em outros países, em nosso estudo este procedimento representou $11,4 \%$ dos casos. Dados da literatura mostram que em geral há maior risco quando o cirurgião tem grande volume cirúrgico ou história prévia de processos médicos ${ }^{(15-17)}$.
Quanto ao tipo de tratamento, cirúrgico ou clínico, nos casos estudados, a cirurgia correspondeu a 94,3\%, sendo a grande maioria associada à facectomia. Estes dados indicam a importância de um cuidadoso preparo pré-operatório, no qual deve ser incluído o desenvolvimento de uma boa relação-médico paciente, o domínio consistente da técnica empregada, condições adequadas de infraestrutura para a realização do procedimento e o correto preenchimento da documentação médica.

Dentre as causas clínicas que originaram a lide, a mais prevalente foi a insatisfação com os óculos prescritos, nesta série, esta foi a motivação de todos os casos não cirúrgicos. Considerando-se que a maior parte dos atendimentos oftalmológicos diários consiste em queixas refrativas, estes resultados indicam a necessidade de um exame refracional cuidadoso, visando evitar insatisfação e risco de processos judiciais.

Quanto às complicações secundárias ao tratamento efetuado, os diagnósticos também indicaram grande associação com resultado cirúrgico, sendo o de maior incidência o descolamento de retina após cirurgia de catarata, seguido de acuidade visual insatisfatória também após cirurgia de catarata e alterações corneanas após cirurgia refrativa. Outros autores relatam resultados similares ${ }^{(10,16-18)}$.

Quanto ao número de consultas prévias ao início do processo, observou-se que a maioria dos casos teve no máximo dois atendimentos antes do fator desencadeante e que após o ocorrido, houve o oposto, com $67,1 \%$ dos pacientes comparecendo a mais de duas consultas. Estes dados parecem indicar que o paciente que teve menor contato com o médico antes do procedimento tem maior probabilidade de entrar com um processo judicial, e que a tentativa de compensar esta deficiência após um procedimento mal sucedido parece não ter efeito no sentido de evitar um processo judicial.

Analisando-se as condições dos exames pré-operatórios de acordo com os protocolos da COOESO, observa-se que um número considerável de casos foi qualificado como insatisfatório (33,3\%) ou até inexistente (3\%). Estes dados são condizentes com o fato de que uma boa avaliação pré-operatória auxilia no diagnóstico da patologia principal e de condições associadas, diminuindo, portanto os riscos operatórios ou servindo ao menos para fornecer uma melhor orientação ao paciente. A avaliação pré-operatória cuidadosa também reforça a relação médico-paciente, na medida em que demonstra o cuidado e atenção dispensados pelo médico. O termo de consentimento informado não foi utilizado em $63,1 \%$ do total de casos cirúrgicos, embora a obrigatoriedade deste documento seja regulamentada por lei. A resolução do Conselho Federal de Medicina, número 1931 de 2009, no capítulo IV sobre direitos humanos, no seu artigo 22 fala que é vedado ao médico deixar de obter consentimento do paciente ou do seu representante legal após esclarecê-lo sobre o procedimento a ser realizado, salvo em caso de risco iminente de morte ${ }^{(19)}$. Esta permissão que deverá ser assinada pelo paciente previamente ao procedimento proposto, é obrigatória, e deverá ter explicações detalhadas sobre os possíveis efeitos colaterais e eventuais resultados adversos baseados em dados da literatura médica, como resultados de grandes séries e consensos.

Analisando-se os processos a maioria resultou em indenização (62,9\%), sendo os valores pagos menores do que 50 mil reais em $72,7 \%$ dos casos, os valores acima de 100 mil reais corresponderam a apenas 4,5\% dos processos. Estas informações indicam que parte destes processos judiciais nem sempre geram prejuízos financeiros consideráveis e raramente atingem altas cifras, apesar disto há o desgaste decorrente da situação e as perdas financeiras indiretas, que poderiam ser evitadas com uma maior atenção à relação médico-paciente, à documentação dos atendimentos e 
procedimentos, e ao correto preenchimento do termo de consentimento informado.

Alguns autores ${ }^{(10)}$,descreveram as principais características de casos judiciais que evoluíram para altos valores indenizatórios nos Estados Unidos. Em concordância com nosso estudo, além da catarata ser o principal diagnóstico, casos que evoluíram para amaurose corresponderam a maior parte dos processos. Ainda de acordo com estes autores, fatores que influenciaram a decisão de procurar a justiça foram registros alterados, pouca documentação e problemas com termos de consentimento. Em nosso estudo, dos 44 casos que apresentaram sentenças desfavoráveis aos médicos, 41 não tinham termo de consentimento. Neste sentido, é importante notar que a defesa médica pode ser afetada por fatores não-clínicos como registros incompletos ou termos de consentimentos inapropriados.

Outros autores ${ }^{(17)}$, em estudo contendo dados a respeito de 120 anos de defesa a processos legais contra oftalmologistas do Reino Unido, indica que a principal causa de processos é a cirurgia de catarata, embora os maiores valores indenizatórios tenham sido relacionados as complicações associadas às cirurgias refrativas.

O código de ética médica de 2009, no capitulo X sobre documentos médicos, descreve no seu artigo 87 que é vedado ao médico deixar de elaborar prontuário legível para cada paciente, e complementa no parágrafo primeiro, que o prontuário deve conter os dados clínicos necessários para boa condução do caso, sendo preenchido, em cada avaliação, em ordem cronológica com data, hora, assinatura e número de registro do médico no Conselho Regional de Medicina(5). É importante enfatizar que a defesa do profissional médico estará fundamentada nos registros contidos no prontuário, no termo de consentimento informado, nas folhas de descrição da cirurgia e em todos os demais documentos pertinentes ao ato que devem estar rigorosamente preenchidos com letra legível e sem rasuras. Portanto, uma boa descrição do caso poderá mostrar nos tribunais que o profissional agiu dentro das normas técnicas, dispensando os cuidados e zelo necessários para obter o melhor resultado para o paciente. Além disso, junto aos autos, no corpo do processo, deverão ser apresentadas provas documentais baseadas em dados da literatura médica que demonstram a possibilidade da complicação ocorrer no procedimento realizado, e que o paciente havia sido informado quando assinou o termo de consentimento.

\section{CONCLUSÃO}

Em suma, foram fatores importantes relacionados aos processos judiciais oftalmológicos, amaurose, tratamento cirúrgi$\mathrm{co}$, descolamento de retina, número reduzido de consultas pré-operatórias e ausência de termo de consentimento.

\section{REFERÊNCIAS}

1. Carvalho JCM. Responsabilidade civil médica. $3^{\underline{a}}$ ed. Rio de Janeiro: Destaque; 2002.

2. Lindstrom R. Lasik and PRK malpractice predictors. Ophthamology. 2004; 111(6):1265-6. Comment in: Ophthalmology. 2003;110(11):2137-46.

3. Deustsche RA, Holzer JF. Cataract surgery associated with majority of malpractice action against ophthalmologists. Ophthalmic Mutual Insurance Company. OMIC Digest [Internet]. 1991. [cited 2010 Jan 12]. Available from: http://www.omic.com/ resources/risk_man/deskref/clinical/1.cfm

4. Donnenfeld ED. Medical malpractice predictors and risk factors for ophthalmologists performing LASIK and photorefractive keratectomy surgery. Evidence-Based Eye Care. 2004;5(2):107-9.

5. Conselho Regional de Medicina do Rio de Janeiro. Código de ética médica: e legislação dos Conselhos de Medicina/ Conselho Regional de Medicina do Rio de Janeiro [Internet]. Rio de Janeiro: CREMERJ; 2010. 80p. [citado 2009 Jun 21]. Disponível em: http://www.cremerj.org.br/publicacoes/133.pdf

6. Couto Filho AF, Souza AP. Responsabilidade civil médica e hospitalar. Belo Horizonte: Del Rey; 2001

7. Panasco WL. A responsabilidade civil, penal e ética dos médicos. Rio de Janeiro: Forense; 1979

8. Santos W. A perícia médica judicial cível, o perito e os assistentes técnicos. In: Nigri AL, Almeida, AHT, editores. Direito e Medicina: um estudo interdisciplinar. Rio de Janeiro: Lumens Juris; 2007. p.253-74.

9. Cooperativa Estadual de Serviços Administrativos em Oftalmologia. Manual de ajustes de conduta [Internet]. Rio de Janeiro: COOESO; 2006.142p. [citado 2009 Nov 20]. Disponível em: http://www.cooeso.com.br/manual.php

10. Krauskar MF, Robb JH. Ophthalmic malpractice lawsuits with large monetary awards. Arch Ophthalmol. 1996;114(3):333-7. Comment in: Arch Ophthalmol. 1996;114(3):339-40.

11. Garcia B, Custódio F. Medicina nos tribunais: um caso cada vez mais comum. Revista DOC. 2009;1 (4):22-7.

12. Férnandez-Vigo J. [Either we protocolize ourselves or they protocolize us: the judicialization of the ophthalmology. Arch Soc Esp Oftalmol. 2007;82(9):533-4. Spanish.

13. Kraushar MF, Turner MF. Medical malpractice litigation in ophthalmology: the New Jersey experience. Ophthalmic Surg. 1986;17(10):671-4.

14. Mavroforou A, Michalodimitrakis E. Physicians'liability in ophthalmology practice. Acta Ophthalmol Scand. 2003;81(4):321-5. Comment in: Acta Ophthalmol Scand. 2003;81(4):319-20.

15. Werre G D. Refractive claims Up, payouts remain low. Ophthalmic Mutual Insurance Company. OMIC Digest [Internet]. 1991. [cited 2009 Jun 12]. Available from: http://www.omic.com/resources/risk_man/deskref/litigation/21.cfm

16. Abbott RL, Ou RJ, Bird M. Medical malpractice predictors and risk factors for ophthalmologists performing LASIK and photorefractive keratectomy surgery. Ophthalmology. 2003;110(11):2137-46. Comment in: Ophthalmology. 2004;111(6): 1265-6; Ophthalmology. 2004;111(6):1263-4; author reply 1264; Ophthalmology. 2004;111(6):1264-5; author reply 1265.

17. Tomkins C. Over 120 years defending ophthalmologists. Brit J Ophtthalmol. 2006: 90(9):1084-5.

18. Kraushar MF. Medical malpractice experiences of vitreoretinal specialists: risk prevention strategies. Retina. 2003;23(4):523-9.

19. Conselho Federal de Medicina. Resolução CFM n.1931/2009. Aprova o Código de Ética Médica [Internet]. Brasilia: CFM; 2010. [citado 2010 Jun 27]. Disponível em: http://portal.cfm.org.br/index.php?option=com content\&view=article\&id= 20670:resolucao-cfm-no-19312009-\&catid=9:codigo-de-etica-medica-atual\& Itemid=122

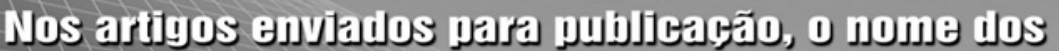

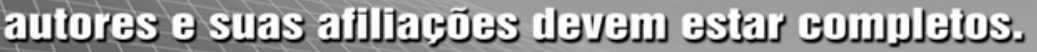

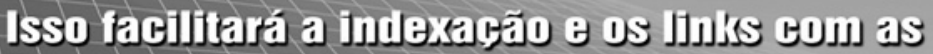

bases do dadus o o CI Lajies. 